\title{
Long non-coding RNA XIST promotes the proliferation of cardiac fibroblasts and the accumulation of extracellular matrix by sponging microRNA-155-5p
}

\author{
HONGBIN ZHANG，JIANFEI MA，FEI LIU and JUN ZHANG \\ Department of Cardiology, Cangzhou Central Hospital, Cangzhou, Hebei 061001, P.R. China
}

Received June 29, 2020; Accepted September 11, 2020

DOI: $10.3892 /$ etm.2021.9908

\begin{abstract}
Acute myocardial infarction (AMI) is characterized by cardiomyocyte death followed by myocardial fibrosis, eventually leading to heart failure. Long non-coding (lnc)RNA X-inactive specific transcript (XIST) serves a vital role in the regulation of fibrosis. The aim of the present study was to determine whether myocardial fibrosis may be regulated by XIST and to elucidate the underlying mechanism. The relative mRNA expression levels of the target genes were evaluated using reverse transcription-quantitative polymerase chain reaction. Cell viability and apoptosis were determined using a Cell Counting Kit-8 assay and flow cytometry, respectively. The apoptosis and fibrosis-related protein expression levels were detected using western blot analysis. Finally, the interaction between XIST and microRNA (miR)-155-5p was analyzed using a luciferase reporter assay. XIST-overexpression increased proliferation and the expression level of the fibrosis-related proteins in the human cardiac fibroblast cells (HCFs). XIST directly targeted miR-155-5p and downregulated its expression, while miR-155-5p downregulation abolished the effect of XIST-silencing on cell viability and the expression level of the fibrosis-related proteins in the HCFs. XIST promoted cell proliferation and the expression level of fibrosis-related proteins by sponging miR-155-5p. Therefore, XIST may represent a novel effective target for AMI treatment.
\end{abstract}

\section{Introduction}

Acute myocardial infarction (AMI) is associated with adverse cardiac remodeling and impaired ventricular function, which is a leading cause of mortality worldwide $(1,2)$. Myocardial

Correspondence to: Dr Jun Zhang, Department of Cardiology, Cangzhou Central Hospital, 16 Xinhua West Road, Cangzhou, Hebei 061001, P.R. China

E-mail: jun_zhang70@126.com

Key words: acute myocardial infarction, X-inactive specific transcript, microRNA-155-5p, myocardial fibrosis, extracellular matrix fibrosis following myocardial infarction is a crucial mechanism of cardiac insufficiency in patients with AMI. A previous study reported that myocardial fibrosis was characterized by the abnormal proliferation of fibroblasts and the accumulation of extracellular matrix (ECM) in the heart, which affected the diastolic and contractile function of the heart, eventually leading to heart failure (HF) $(3,4)$. Due to the adverse impact of AMI on the survival rate of patients, developing novel therapeutic strategies to improve cardiac performance by reducing the number of myocardial fibroblasts following AMI are desirable.

Long non-coding (lnc)RNAs are a type of RNA transcript, longer than 200 nucleotides in length and with no open reading frame(5). A previous study suggested that lncRNAs serve important roles in diverse biological processes, and their vital role in the regulation of fibrosis is increasingly being elucidated (6). $\mathrm{X}$-inactive specific transcript (XIST) was highly expressed in hypertrophic mouse hearts and promoted the progression of cardiac hypertrophy by targeting microRNA(miR)-101 to upregulate TLR2 expression (7). However, the biological roles of IncRNA XIST in myocardial injury and fibrosis have not been widely reported. McKiernan et al (8) reported that XIST was aberrantly expressed in cystic fibrosis bronchial epithelium in vivo, which may be associated with inflammation. Furthermore, XIST promoted bleomycin-induced pulmonary fibrosis by inhibiting miR-139 to enhance the proliferative ability of human/mouse fibroblasts and ECM protein expression (9). Notably, XIST-silencing may protect against renal interstitial fibrosis in diabetic nephropathy by miR-93-5p-dependent CDKN1A inhibition (10). Therefore, we hypothesized that XIST may contribute toward myocardial fibroblasts following AMI.

Angiotensin II (Ang II) is the main active protein in the rennin-angiotensin-aldosterone system (RAAS), which serves a crucial role in myocardial interstitial fibrosis and cardiac remodeling $(11,12)$. Under pathological conditions, Ang II stimulates the proliferation of cardiac fibroblast cells (CFs) by autocrine and paracrine signaling, and promotes the synthesis of collagen (Col) I to repair injury in myocardial tissues (13-15). However, the continuous abnormal synthesis of Col I, caused by the excessive proliferation of CFs, may lead toward myocardial interstitial fibrosis and decreased compliance of the ventricle, which ultimately affects the diastolic and contractile function of the heart. 
In the present study, human cardiac fibroblast cells (HCFs) were used and treated with Ang II, while pcDNA3.1 XIST and short hairpin (sh)RNA-XIST was used to induce XIST overexpression and silencing in cells, respectively. The present study was designed to determine whether myocardial fibrosis could be regulated by XIST and to elucidate the related mechanism. The results demonstrated that XIST promoted the proliferation of myocardial fibroblasts and the accumulation of ECM, suggesting that XIST may be a novel potential target for AMI treatment.

\section{Materials and methods}

Cell culture and transfection. Primary HCFs were obtained from the American Type Culture Collection and were maintained in Dulbecco's modified Eagle's medium (DMEM, Hyclone; Cytival), supplemented with 10\% FBS (Invitrogen; Thermo Fisher Scientific, Inc.) and $1 \%$ penicillin and $1 \%$ streptomycin (Gibco; Thermo Fisher Scientific, Inc.) at $37^{\circ} \mathrm{C}$ for $48 \mathrm{~h}$ in a humidified incubator with $5 \% \mathrm{CO}_{2}$, followed by Ang II (Sigma-Aldrich; Merck KGaA) treatment (0.01 mM) at $37^{\circ} \mathrm{C}$ for $24 \mathrm{~h}$ to induce the myocardial fibrosis phenotype.

At $70-80 \%$ confluence, HCFs were seeded onto a corresponding culture plate and starved for $12 \mathrm{~h}$ in serum-free DMEM (Hyclone; Cytival) prior to treatment. The plasmids, including $1 \mu \mathrm{g} /$ well pcDNA3.1 XIST (forward, 5'-CCAAGC TTTGCACACGGCCTATCTCATC-3' and reverse, 5'-CCG CTCGAGTGAAAAGAGGTGGGGCATCC-3') inserted into pcDNA3.1, 100 pmol of short hairpin RNA (shRNA)-XIST (shRNA-XIST-1, 5'-TTC TCCGAACGTGTCACGT-3'; 100 pmol of shRNA-XIST-2, 5'-GCTGCTAGTTTCCCAATG ATA-3'), 100 pmol of shRNA-NC (5'-TTCTCCGAACGTGTC ACGT-3'), $20 \mathrm{nM}$ of miR-155-5p mimic (forward, 5'-UUAAUG CUAAUCGUGAUAGGGGU-3' and reverse, 5'-CCCUAU CACGAUUAGCAUUAAUU-3'), $20 \mathrm{nM}$ of mimic-negative control (NC; forward, 5'-UUCCUCCGAACGUGUCACGUT T-3' and reverse, 5'-ACGUGACACGUUCGGAGAATT-3'), $100 \mathrm{nM}$ of miR-155-5p inhibitor (5'-ACCCCUAUCACGAUU AGCAUUAA-3') and $100 \mathrm{nM}$ of inhibitor-NC (5'-CAGUAC UUUUGUGUAGUACAA-3') were purchased from Shanghai GenePharma Co., Ltd., and transfected into the HCFs using Lipofectamine $^{\circledR} 3000$ (Invitrogen; Thermo Fisher Scientific, Inc.) according to the manufacturer's protocols. The interaction between XIST and miR-155-5p was predicted using the Starbase website (http://starbase.sysu.edu.cn/index.php). After $48 \mathrm{~h}$, the HCFs were used for further experiments and reverse transcription-quantitative polymerase chain reaction (RT-qPCR) was performed to examine transfection efficiency.

$R T$ - $q P C R$. Total RNA was extracted from the HCFs using TRIzol $^{\circledR}$ (Invitrogen; Thermo Fisher Scientific, Inc.) according to the manufacturer's protocols. First strand cDNA was synthesized at $42^{\circ} \mathrm{C}$ for $60 \mathrm{~min}$ and $95^{\circ} \mathrm{C}$ for $5 \mathrm{~min}$, using a PrimeScript RT reagent kit. qPCR was performed using a $\mathrm{SYBR}^{\circledR}$ Green Real-Time PCR Master Mix (Takara Bio, Inc.). qPCR amplification parameters were: Annealing at $25^{\circ} \mathrm{C}$ for $10 \mathrm{~min}$, extension at $42^{\circ} \mathrm{C}$ for $30 \mathrm{~min}$; inactivation at $85^{\circ} \mathrm{C}$ for $10 \mathrm{~min}$, after extension of the detection of fluorescence signals, a total of 40 cycles. The $2^{-\Delta \Delta \mathrm{Cq}}$ method (16) was used to quantify the relative mRNA expression of the target and housekeeping genes. The following primers were used: XIST forward, 5'-ACGCTGCATGTGTCCTTAG-3 and reverse, 5'-GAGCCTCTTATAAGCTGTTTG-3'; miR-155-5p forward, 5'-GAGGGTTAATGCTAATCGTGATAGG-3' and reverse, 5'-GCACAGAATCAACACGACTCACTAT-3'; GAPDH forward, 5'-CTGGGCTACACTGAGCACC-3' and reverse, 5'-AAGTGGTCGTTGAGGGCAATG-3'; and U6 forward, 5'-GAGGGTTAATGCTAATCGTGATAGG-3' and reverse, 5'-GCACAGAATCAACACGACTCACTAT-3'.

Cell proliferation. After the HCFs $\left(4 \times 10^{3} /\right.$ well) from different experimental groups, were plated onto 96 -well plates and cultured for $24 \mathrm{~h}$, the Cell Counting Kit-8 (CCK-8; Dojindo Molecular Technologies, Inc.) was added (10 $\mu \mathrm{l} /$ well) and incubated with the HCFs for another $2 \mathrm{~h}$ according to manufacturer's protocol. A microplate reader was used to detect the absorbance at $450 \mathrm{~nm}$. The experiment was repeated independently three times.

Flow cytometry. The Annexin V-FITC/PI apoptosis detection kit (Beyotime Institute of Biotechnology) was used according to the manufacturer's protocols. The harvested cells $\left(1 \times 10^{5}\right.$ cells $\left./ \mathrm{ml}\right)$ were resuspended in $500 \mu \mathrm{l}$ of $1 \mathrm{X}$ binding buffer (Beyotime Institute of Biotechnology). Next, the cells were incubated with $5 \mu \mathrm{l}$ Annexin V-FITC followed by $10 \mu \mathrm{l}$ PI staining solution. Following incubation in the dark, at room temperature for $20 \mathrm{~min}$, the fluorescence was detected using a flow cytometer (FACScan ${ }^{\mathrm{TM}}$; BD Biosciences) and ModFit software (version 3.2; Verity Software House, Inc.) to analyze the apoptotic rate of HCFs.

Western blot analysis. The HCFs in each group were lysed with RIPA lysis buffer (Beyotime Institute of Biotechnology). Total protein was determined using a bicinchoninic acid assay (Thermo Fisher Scientific, Inc.). Next, the protein samples $(25 \mu \mathrm{g})$ were separated using 10\% SDS-PAGE, transferred to polyvinylidene difluoride membranes, and blocked with $5 \%$ skimmed milk at room temperature for $1 \mathrm{~h}$. The membranes were incubated overnight at $4{ }^{\circ} \mathrm{C}$ with the primary antibodies against Bcl-2 (1:1,000; cat. no. 2875; Cell Signaling Technology, Inc.), Bax (1:1,000; cat. no. 2772, Cell Signaling Technology, Inc.), cleaved-caspase-9 (1:500; cat. no. 9509; Cell Signaling Technology, Inc.), cleaved caspase-3 (1:500; cat. no. 9654; Cell Signaling Technology, Inc.), caspase-3 (1:1,000; cat. no. 9668; Cell Signaling Technology, Inc.), caspase-9 (1:1,000; cat. no. 9502; Cell Signaling Technology, Inc.), Col I (1:1,000; ab34710; Abcam), Col III (1:1,000; ab6310; Abcam), $\alpha$-smooth muscle actin ( $\alpha$-SMA; 1:1,000; cat. no. 68463; Cell Signaling Technology, Inc.), cytochrome C (1:1,000; cat. no. 12959; Cell Signaling Technology, Inc.), cleaved PARP (1:500; cat.no.9185; Cell Signaling Technology, Inc.), and PARP (1:1,000; cat. no. 9542; Cell Signaling Technology, Inc.). Next, the membranes were incubated with horseradish peroxidase-conjugated horseradish peroxidase-conjugated anti-rabbit IgG secondary antibodies (1:1,000; cat. no. 7074; Cell Signaling Technology, Inc.) at room temperature for $2 \mathrm{~h}$ and visualized using a Tanon-5200 chemiluminescence imager (Tanon Science and Technology Co., Ltd) using an enhanced chemiluminescence kit and the intensity of the bands was normalized to GAPDH expression. 


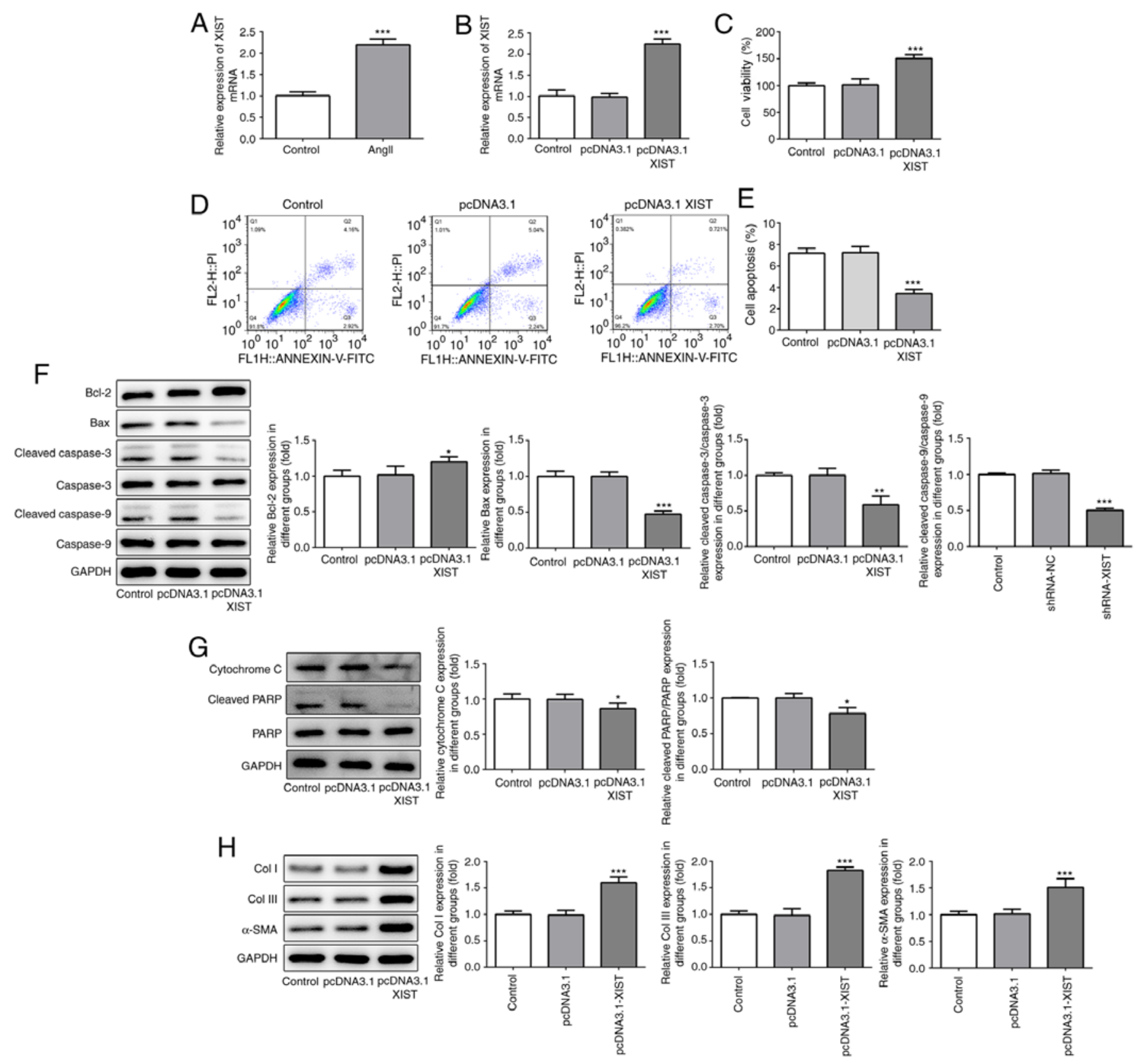

Figure 1. XIST-overexpression promoted cell proliferation and increased the expression of fibrosis-related proteins in the HCFs. (A and B) The relative mRNA expression level of XIST was determined using reverse transcription-quantitative polymerase chain reaction. (C) The viability of the HCFs was analyzed using a Cell Counting Kit-8 assay. (D and E) The apoptotic rates of the HCFs transfected with or without pcDNA3.1 XIST were determined using flow cytometry. The protein expression of (F) Bcl-2, Bax, cleaved cacpase-3, cacpase-3, cleaved cacpase-9, cacpase-9; (G) cytochrome $c$, cleaved PARP and PARP; and $(\mathrm{H}) \mathrm{Col}$ I, Col III and $\alpha$-SMA were detected using western blot analysis. The data are expressed as the mean \pm standard error of the mean, from three independent experiments. ${ }^{*} \mathrm{P}<0.05,{ }^{* * *} \mathrm{P}<0.01,{ }^{* * * *} \mathrm{P}<0.001$ vs. control. Col, collagen; $\alpha$-SMA, $\alpha$ smooth muscle actin; HCFs, human cardiac fibroblast cells.

Luciferase reporter assay. The HCFs $\left(1 \times 10^{5}\right)$ were seeded onto 24-well plates and cultured for $24 \mathrm{~h}$. The luciferase reporter vector for the wild-type (WT) or mutant (MUT) type 3'-untranslated region (UTR) of XIST, which contained the binding sites between miR-155-5p was cloned into the pMIR-reporter luciferase system (Thermo Fisher Scientific, Inc.). The MUT 3'-UTR of XIST was constructed using the QuikChang Site-Directed Mutagenesis kit (Agilent Technologies). miR-155-5p mimic or mimic-NC combined with the vector expressing firefly luciferase reporter fused with WT or MUT 3'-UTR of XIST was co-transfected into the HCFs using Lipofectamine ${ }^{\circledR} 2000$ (Invitrogen; Thermo Fisher
Scientific, Inc.). Following incubation for $24 \mathrm{~h}$, the HCFs were collected and the luciferase activities were determined using the dual-luciferase reporter assay system (Promega Corporation) and the firefly luciferase activity was normalized to Renilla luciferase activity.

Statistical analysis. The data are presented as the mean \pm standard error of the mean and analyzed using GraphPad Prism v5.0 (GraphPad Software, Inc.). The difference between two groups was determined using a two-tailed Student's t-test, while a one-way analysis of variance, followed by Bonferroni's post hoc test, was used for multiple 

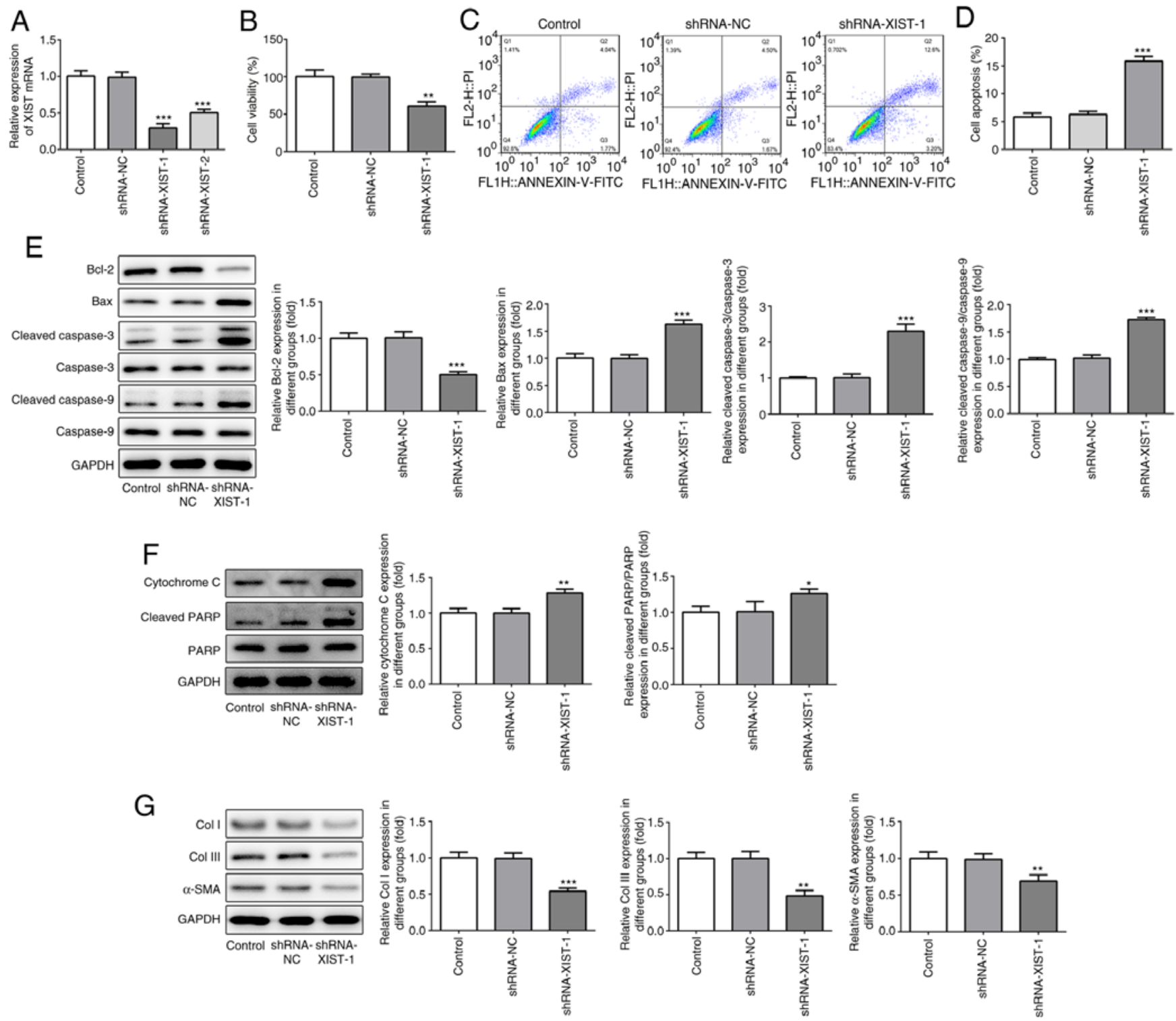

Figure 2. XIST-silencing inhibited the proliferation and expression of fibrosis-related proteins in the HCFs. (A) The relative mRNA expression level of XIST was determined using reverse transcription-quantitative polymerase chain reaction. (B) The viability of the HCFs was analyzed using Cell Counting Kit-8 assay. (C) The apoptotic rate of the HCFs transfected with or without shRNA-XIST-1 was determined using flow cytometry and was (D) subsequently quantified. The protein expression of (E) Bcl-2, Bax, cleaved cacpase-3, cacpase-3, cleaved cacpase-9, cacpase-9; (F) cytochrome $c$, cleaved PARP and PARP; and $(\mathrm{G}) \mathrm{Col}$ I, Col III and $\alpha$-SMA was detected using western blot analysis. The data are presented as the mean \pm standard error of the mean, from three independent experiments. ${ }^{*} \mathrm{P}<0.05,{ }^{* *} \mathrm{P}<0.01,{ }^{* * *} \mathrm{P}<0.001$ vs. control. Col, collagen; $\alpha$-SMA, $\alpha$ smooth muscle actin; sh, short hairpin; NC, negative control; HCFs, human cardiac fibroblast cells.

groups. $\mathrm{P}<0.05$ was considered to indicate a statistically significant difference.

\section{Results}

XIST overexpression promotes proliferation and the protein expression level of fibrosis-related proteins in HCFs. To investigate the association between XIST and myocardial fibrosis, RT-qPCR was performed to detect XIST mRNA expression following treatment of the HCFs with Ang II. As shown in Fig. 1A, Ang II treatment significantly increased the relative mRNA expression of XIST, compared with that in the control group. Subsequently, the pcDNA3.1-XIST plasmid was used to induce XIST overexpression, which was confirmed by the RT-qPCR results (Fig. 1B). The CCK-8 assay was performed to analyze the cell viability of the HCFs. As shown in Fig. 1C, the HCFs in the pcDNA3.1-XIST group had a higher rate of viability, compared with that in the control and pcDNA3.1 groups. Furthermore, cell apoptosis was detected using flow cytometry and the protein expression level of the apoptosis-related proteins were quantified using western blot analysis. As shown in Fig. 1D and E, the apoptotic rate was significantly decreased in the pcDNA3.1-XIST group, compared with that in the control group. In addition, XIST-overexpression induced upregulation of Bcl-2, while Bax, cleaved caspase-3 and -9 , cytochrome $c$ and cleaved PARP were downregulated (Fig. 1F and G). Finally, the protein expression levels of Col I, Col III and $\alpha$-SMA were also determined using western blot analysis and the results revealed that the expression of these proteins was significantly increased by 

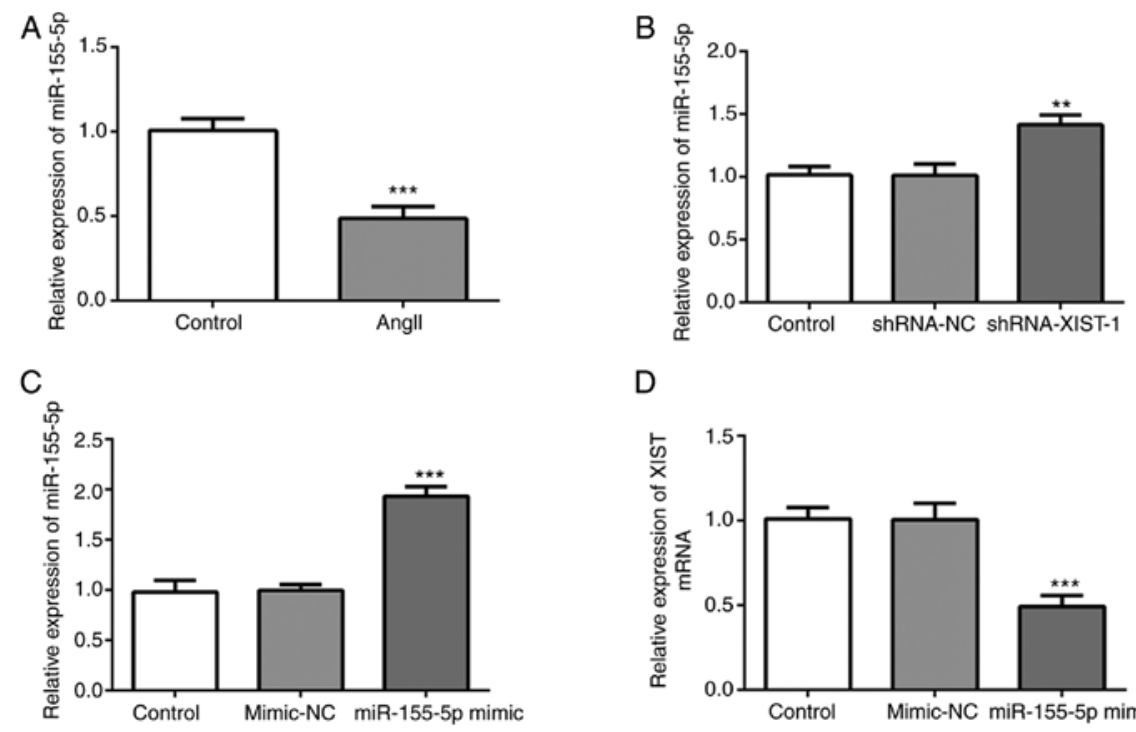

D

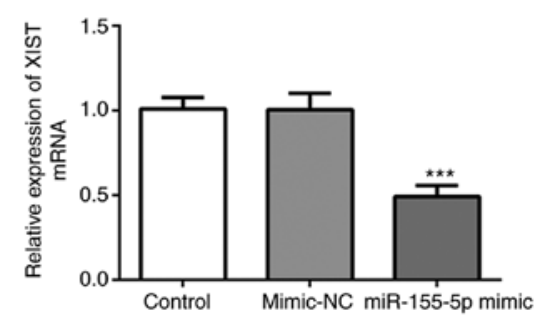

$E$

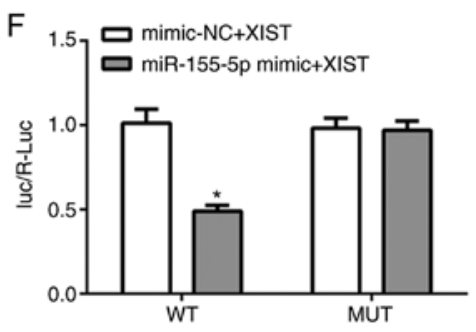

Figure 3. XIST directly targets miR-155-5p and downregulates its expression. (A-C) Relative mRNA expression of miR-155-5p was determined using RT-qPCR. (D) Relative mRNA expression of XIST was determined using RT-qPCR. (E) The binding sites between XIST and miR-155-5p. (F) The relative luciferase activities in the human cardiac fibroblast cells were evaluated using a luciferase reporter assay. The data are presented as the mean \pm standard error of the mean, from three independent experiments. ${ }^{*} \mathrm{P}<0.05,{ }^{* *} \mathrm{P}<0.01,{ }^{* * * *} \mathrm{P}<0.001$ vs. control. miR, microRNA; RT-qPCR, reverse transcription-quantitative polymerase chain reaction; WT, wild-type; MUT, mutant; NC, negative control; Ang II, angiotensin II; sh, short hairpin.

XIST overexpression (Fig. 1H). These results suggested that XIST-overexpression promoted the proliferation of HCFs and the expression of the fibrosis-related proteins in vitro.

XIST-silencing inhibits proliferation and decreases the expression of fibrosis-related proteins in HCFs. To confirm the role of XIST in myocardial fibrosis, shRNA-XIST-1 and shRNA-XIST-2 were used to induce XIST-silencing. As shown in Fig. 2A, the RT-qPCR results demonstrated that a lower mRNA expression level of XIST was observed in the HCFs in the shRNA-XIST-1 group, compared with that in the shRNA-XIST-2 group; therefore, shRNA-XIST-1 was selected in the following experiments. The CCK- 8 assay results demonstrated that XIST-silencing significantly suppressed cell viability of the HCFs (Fig. 2B). The flow cytometry results revealed that XIST-silencing significantly enhanced the apoptotic rate of the HCFs (Fig. 2C and D). In addition, XIST-silencing increased the protein expression level of Bax, cleaved caspase-3 and -9, cytochrome $c$ and cleaved PARP, while the protein expression level of Bcl-2, Col I, Col III and $\alpha$-SMA was decreased (Fig. 2E-G). These results indicated that XIST-silencing inhibited proliferation of the HCFs and the protein expression level of fibrosis-related proteins in vitro.

XIST directly targets miR-155-5p and downregulates its $m R N A$ expression level. To investigate the specific mechanism of XIST in myocardial fibrosis, RT-qPCR was performed to detect miR-155-5p mRNA expression, following treatment with Ang II. As shown in Fig. 3A, Ang II treatment significantly decreased the relative mRNA expression of miR-155-5p. In addition, the RT-qPCR results demonstrated that the mRNA expression of miR-155-5p could be increased by XIST-silencing (Fig. 3B). Subsequently, miR-155-5p mimic was used to induce miR-155-5p-overexpression. The RT-qPCR results demonstrated that miR-155-5p mimic was transfected into the cells successfully and upregulated miR-155-5p mRNA expression (Fig. 3C). Notably, XIST expression was significantly decreased by miR-155-5p-overexpression (Fig. 3D). The interaction between XIST and miR-155-5p was predicted using the Starbase website (http://starbase.sysu.edu.cn/index. php), as presented in Fig. 3E. Subsequently, the luciferase reporter assay was performed to confirm whether miR-155-5p was a direct target of XIST. As shown in Fig. 3F, the relative luciferase activity was significantly lower in the HCFs co-transfected with WT-XIST and miR-155-5p mimic than that in the control group. These results indicated that XIST specifically bound to the 3'-UTR of miR-155-5p to decrease its expression.

miR-155-5p-downregulation abolishes the effect of XIST-silencing on cell viability and the protein expression level of the fibrosis-related proteins in the HCFs. To further investigate the molecular mechanism of XIST in myocardial fibrosis, the HCFs were transfected with an miR-155-5p inhibitor. As 
A

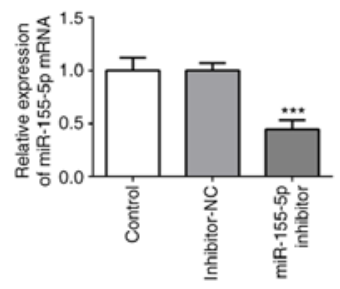

B

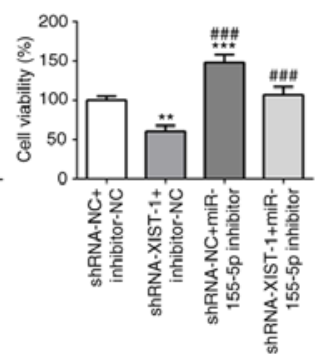

$\mathrm{E}$

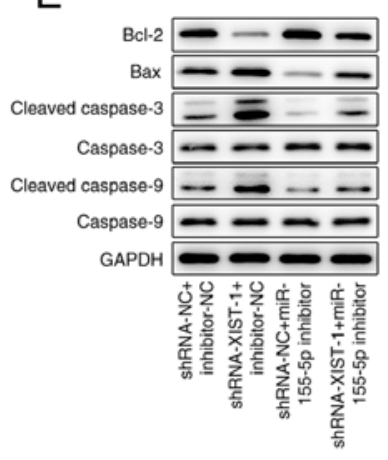

$\mathrm{F}$

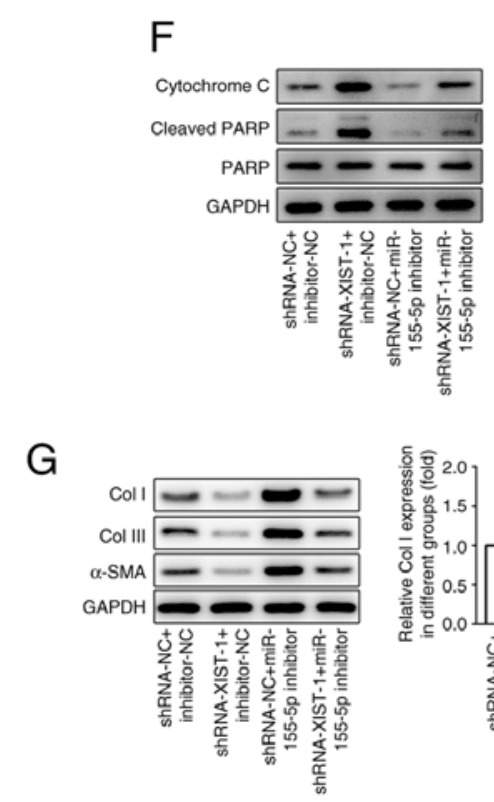

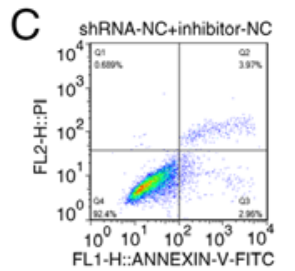

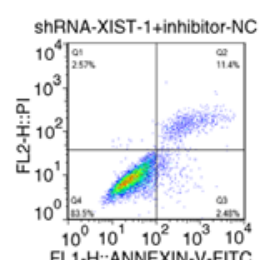

ShRNA-NC-1+miR-155-5p inhibitor shRNA-XIST-1+miR-155-5p inhibitor
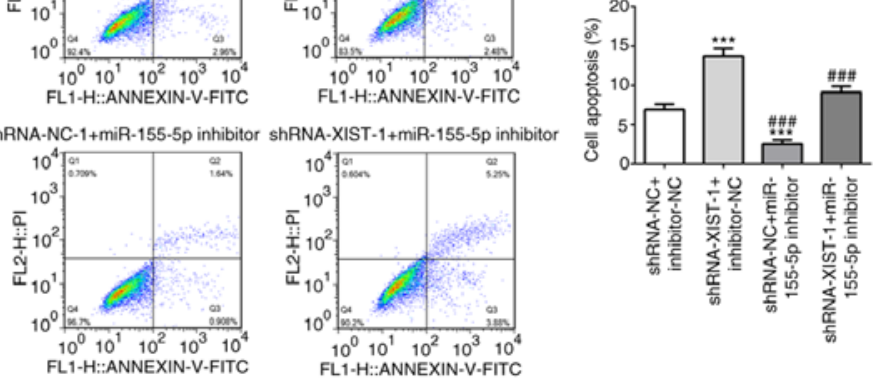
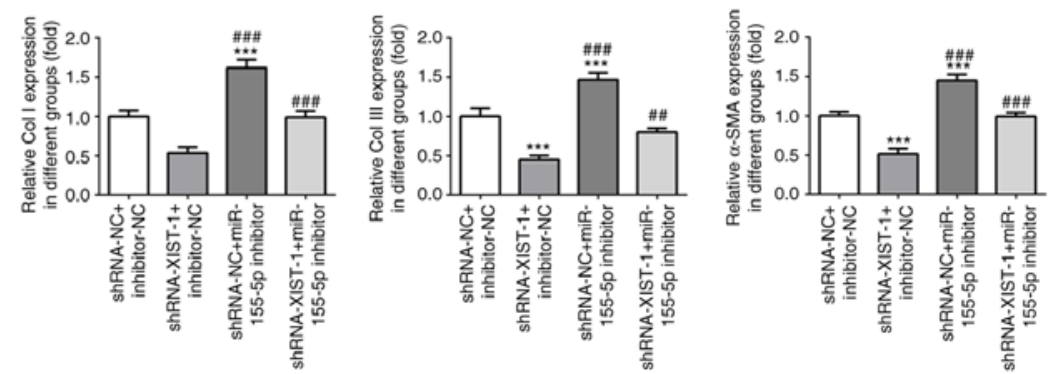

Figure 4. miR-155-5p-downregulation abolished the effect of XIST-silencing on cell viability and the expression of fibrosis-related proteins in the HCFs. (A) The relative mRNA of miR-155-5p was determined by reverse transcription-quantitative polymerase chain reaction. (B) The viability of the HCFs was analyzed using a Cell Counting Kit-8 assay. (C) The apoptotic rate of the HCFs in each group was determined using flow cytometry and was (D) subsequently quantified. The protein expression of (E) Bcl-2, Bax, cleaved caspase-3, caspase-3, cleaved-caspase-9, caspase-9; (F) cytochrome $c$, cleaved PARP and PARP; and $(\mathrm{G}) \mathrm{Col}$ I, Col III and $\alpha$-SMA was detected using western blot analysis. The data are presented as the mean \pm standard error of the mean, from three independent experiments. ${ }^{*} \mathrm{P}<0.05,{ }^{* *} \mathrm{P}<0.01,{ }^{* * *} \mathrm{P}<0.001$ vs. shRNA-NC+inhibitor-NC. ${ }^{\# \#} \mathrm{P}<0.05,{ }^{\# \# \#} \mathrm{P}<0.001$ vs. shRNA-XIST-1+inhibitor-NC. miR, microRNA; HCFs, human cardiac fibroblast cells; sh, short hairpin; NC, negative control; Col, collagen; $\alpha$-SMA, $\alpha$ smooth muscle actin.

shown in Fig. 4A, miR-155-5p-downregulation was achieved via miR-155-5p inhibitor transfection. The CCK-8 assay results demonstrated that miR-155-5p inhibitor abolished the suppressive effects of XIST-silencing on cell viability (Fig. 4B). The flow cytometry results demonstrated that XIST-silencing promoted apoptosis in the HCFs, which was partly reversed by miR-155-5p downregulation (Fig. 4C and D). The western blot result showed that miR-155-5p downregulation abolished the regulatory effect of XIST on the protein expression level of the apoptosis-related proteins (Fig. 4E and F), as well as the fibrosis-related proteins (Fig. 4G). These results suggested that XIST promoted the proliferation of the HCFs and the 
expression level of the fibrosis-related proteins by sponging miR-155-5p.

\section{Discussion}

AMI is one of the diseases with the highest morbidity and mortality rates in the world (17), and it may induce cardiac remodeling and HF (18). Myocardial fibrosis has been reported to be associated with sparseness of cardiac microvessels, and found to contribute toward HF, which is caused by myocardial fibroblast accumulation and imbalance of synthesis, metabolism and degradation of collagen in ECM deposition (19). CFCs are the primary cells that secrete the ECM of the myocardium. In the present study, HCFs were treated with Ang II to induce the myocardial fibrosis phenotype. Notably, it was observed that XIST was highly expressed in the HCFs treated with Ang II, suggesting that XIST was associated with the onset and development of myocardial fibrosis.

A recent study demonstrated that XIST was upregulated in ethanol-induced human hepatic stellate cells and contributed toward liver fibrogenesis (20), which was consistent with the results of the present study. Furthermore, the results of the present study suggested that XIST-overexpression promoted proliferation and enhanced the protein expression of fibrosis-related proteins in HCFs, while XIST-silencing had the opposite effect. These data indicated that XIST may promote the development of myocardial fibrosis.

To investigate the specific mechanisms underlying the role of XIST in myocardial fibrosis, the interaction between XIST and miR-155-5p was predicted using the Starbase website. It was found that miR-155-5p was one of the potential targets of XIST. In the present study, Ang II treatment led to a decrease in miR-155-5p mRNA expression, whereas XIST downregulation significantly increased miR-155-5p mRNA expression. These data suggested that XIST may directly target miR-155-5p and downregulate its mRNA expression level. A recent study has demonstrated that miR-155-5p suppressed the proliferation, migration and invasion of vascular smooth muscle cells (VSMCs) and human umbilical vein endothelial cells in atherosclerosis by regulating Akt (21). Chen et al (21) reported that miR-155-5p inhibition prevented the development of abdominal aortic aneurysms in mice by regulating macrophage-mediated inflammation (22). It was reported that miR-155-overexpression decreased cellular proliferation in VSMCs, and the expression of miR-155 was decreased and negatively correlated with calcification in the aorta of chronic kidney disease rats (23). Additionally, a previous study reported that miR-155-5p mediated the regulatory network for myocardial infarction (24), suggesting that miR-155-5p may be a putative therapeutic target for AMI. In addition, miR-155-5p-upregulation decreased vascular thickening and fibrosis in a vascular calcification model (25). In the present study, miR-155-5p-downregulation promoted the proliferation of the HFCs and suppressed cell apoptosis. It is well-known that collagen, an insoluble fibrous protein, is the main component of the ECM $(26,27)$. miR-155-5p downregulation abolished the inhibitory effect of XIST-silencing on the protein expression level of Col I, Col III and $\alpha$-SMA in the HCFs, suggesting that XIST promoted the proliferation of the HCFs and the protein expression of fibrosis-related proteins by sponging miR-155-5p, as shown in Fig. S1.

In summary, XIST-silencing inhibited the proliferation of the HCFs and collagen expression, which was partially reversed by miR-155-5p-downregulation; therefore, XIST promoted cell proliferation and the protein expression of the fibrosis-related proteins by sponging miR-155-5p. Therefore, XIST may be a novel effective target for AMI treatment.

\section{Acknowledgements}

Not applicable.

\section{Funding}

No funding was received.

\section{Availability of data and materials}

The datasets used and/or analyzed during the current study are available from the corresponding author on reasonable request.

\section{Authors' contributions}

JZ designed the study. HZ and JM were responsible for the data collection and analysis. HZ and FL collaborated to perform the data analysis. All authors collaborated to interpret results and develop the manuscript. All authors read and approved the final version of the manuscript.

\section{Ethics approval and consent for participation}

Not applicable.

\section{Patient consent for publication}

Not applicable.

\section{Competing interests}

The authors declare that they have no competing interests.

\section{References}

1. Davidson SM, Ferdinandy P, Andreadou I, Bøtker HE, Heusch G, Ibáñez B, Ovize M, Schulz R, Yellon DM, Hausenloy DJ, et al: Multitarget strategies to reduce myocardial ischemia/reperfusion injury: JACC review topic of the week. J Am Coll Cardiol 73: 89-99, 2019.

2. Kurose $\mathrm{H}$ and Mangmool S: Myofibroblasts and inflammatory cells as players of cardiac fibrosis. Arch Pharm Res 39: 1100-1113, 2016.

3. Cho N, Razipour SE and McCain ML: Featured Article: TGF- $\beta 1$ 1 dominates extracellular matrix rigidity for inducing differentiation of human cardiac fibroblasts to myofibroblasts. Exp Biol Med (Maywood) 243: 601-612, 2018.

4. Yuan X, Pan J, Wen L, Gong B, Li J, Gao H, Tan W, Liang S, Zhang $\mathrm{H}$ and Wang X: MiR-144-3p enhances cardiac fibrosis after myocardial infarction by targeting PTEN. Front Cell Dev Biol 7: 249, 2019

5. Puvvula PK: LncRNAs regulatory networks in cellular senescence. Int J Mol Sci 20: 2615, 2019. 
6. Yarani R, Mirza AH, Kaur S and Pociot F: The emerging role of lncRNAs in inflammatory bowel disease. Exp Mol Med 50: $1-14,2018$.

7. Xiao L, Gu Y, Sun Y, Chen J, Wang X, Zhang Y, Gao L and Li L: The long noncoding RNA XIST regulates cardiac hypertrophy by targeting miR-101. J Cell Physiol 234: 13680-13692, 2019.

8. McKiernan PJ, Molloy K, Cryan SA, McElvaney NG and Greene CM: Long noncoding RNA are aberrantly expressed in vivo in the cystic fibrosis bronchial epithelium. Int $\mathrm{J}$ Biochem Cell Biol 52: 184-191, 2014.

9. Wang Y, Liang Y, Luo J, Nie J, Yin H, Chen Q, Dong J, Zhu J, Xia J and Shuai W: XIST/miR-139 axis regulates bleomycin (BLM)-induced extracellular matrix (ECM) and pulmonary fibrosis through $\beta$-catenin. Oncotarget 8: 65359-65369, 2017.

10. Yang J, Shen Y, Yang X, Long Y, Chen S, Lin X, Dong R and Yuan J: Silencing of long noncoding RNA XIST protects against renal interstitial fibrosis in diabetic nephropathy via microRNA-93-5p-mediated inhibition of CDKN1A. Am J Physiol Renal Physiol 317: F1350-F1358, 2019.

11. Liu Y, Gao L, Guo S, Zhao X, Li R, Yan X, Li Y, Wang S, Niu X, Yao L, et al: Kaempferol alleviates angiotensin II-induced cardiac dysfunction and interstitial fibrosis in mice. Cell Physiol Biochem 43: 2253-2263, 2017.

12. Wang ZF, Wang NP, Harmouche S, Philip T, Pang XF, Bai F and Zhao ZQ: Postconditioning attenuates coronary perivascular and interstitial fibrosis through modulating angiotensin II receptors and Angiotensin-converting enzyme 2 after myocardial infarction. J Surg Res 211: 178-190, 2017.

13. Lin C, Wei D, Xin D, Pan J and Huang M: Ellagic acid inhibits proliferation and migration of cardiac fibroblasts by Down-regulating expression of HDAC1. J Toxicol Sci 44: 425-433, 2019.

14. Tang CM, Zhang M, Huang L, Hu ZQ, Zhu JN, Xiao Z, Zhang Z, Lin QX, Zheng XL, Yang M, et al: CircRNA_000203 enhances the expression of fibrosis-associated genes by derepressing targets of miR-26b-5p, Colla2 and CTGF, in cardiac fibroblasts. Sci Rep 7: 40342, 2017.

15. Zhao N, Yu H, Sun M, Zhang Y, Xu M and Gao W: MiRNA-711-SP1-collagen-I pathway is involved in the anti-fibrotic effect of pioglitazone in myocardial infarction. Sci China Life Sci 56: 431-439, 2013.

16. Maruyama T, Nishihara K, Umikawa M, Arasaki A, Nakasone T, Nimura F, Matayoshi A, Takei K, Nakachi S, Kariya KI, et al: MicroRNA-196a-5p is a potential prognostic marker of delayed lymph node metastasis in early-stage tongue squamous cell carcinoma. Oncol Lett 15: 2349-2363, 2018.

17. Castro-Dominguez Y, Dharmarajan K and McNamara RL: Predicting death after acute myocardial infarction. Trends Cardiovasc Med 28: 102-109, 2018.
18. Yin Y, Zhang Q, Zhao Q, Ding G, Wei C, Chang L, Li H, Bei H, Wang $\mathrm{H}$, Liang $\mathrm{J}$, et al: Tongxinluo attenuates myocardiac fibrosis after acute myocardial infarction in rats via inhibition of endothelial-to-mesenchymal transition. Biomed Res Int 2019: 6595437, 2019.

19. van Putten S, Shafieyan Y and Hinz B: Mechanical control of cardiac myofibroblasts. J Mol Cell Cardiol 93: 133-142, 2016.

20. Xie ZY, Wang FF, Xiao ZH, Liu SF, Lai YL and Tang SL: Long noncoding RNA XIST enhances ethanol-induced hepatic stellate cells autophagy and activation via miR-29b/HMGB1 axis. IUBMB Life 71: 1962-1972, 2019.

21. Chen L, Zheng SY, Yang CQ, Ma BM and Jiang D: MiR-155-5p inhibits the proliferation and migration of VSMCs and HUVECs in atherosclerosis by targeting AKT1. Eur Rev Med Pharmacol Sci 23: 2223-2233, 2019.

22. Zhang Z, Liang K, Zou G, Chen X, Shi S, Wang G, Zhang K, $\mathrm{Li} \mathrm{K}$ and Zhai S: Inhibition of miR-155 attenuates abdominal aortic aneurysm in mice by regulating macrophage-mediated inflammation. Biosci Rep 38: BSR20171432, 2018.

23. Chen NX, Kiattisunthorn K, O'Neill KD, Chen X, Moorthi RN, Gattone VH II, Allen MR and Moe SM: Decreased microRNA is involved in the vascular remodeling abnormalities in chronic kidney disease (CKD). PLoS One 8: e64558, 2013.

24. Zhang G, Shi H, Wang L, Zhou M, Wang Z, Liu X, Cheng L, $\mathrm{Li} \mathrm{W}$ and Li X: MicroRNA and transcription factor mediated regulatory network analysis reveals critical regulators and regulatory modules in myocardial infarction. PLoS One 10: $\mathrm{e} 0135339,2015$.

25. Zhao F, Wu Y, Yang W, Wu D, Wang C and Zhang F: Inhibition of vascular calcification by microRNA-155-5p is accompanied by the inactivation of TGF- $\beta 1 / \mathrm{Smad} 2 / 3$ signaling pathway. Acta Histochem 122: 151551, 2020

26. Halper J and Kjaer M: Basic components of connective tissues and extracellular matrix: Elastin, fibrillin, fibulins, fibrinogen, fibronectin, laminin, tenascins and thrombospondins. Adv Exp Med Biol 802: 31-47, 2014

27. Jabłońska-Trypuć A, Matejczyk M and Rosochacki S: Matrix metalloproteinases (MMPs), the main extracellular matrix (ECM) enzymes in collagen degradation, as a target for anticancer drugs. J Enzyme Inhib Med Chem 31 (Suppl 1): S177-S183, 2016 .

c) () $(-)$ This work is licensed under a Creative Commons

Attribution-NonCommercial-NoDerivatives 4.0 International (CC BY-NC-ND 4.0) License. 